\title{
Progress on China nuclear data processing code system
}

\author{
Ping Liu ${ }^{1, \text { a }}$, Xiaofei $\mathrm{Wu}^{1}$, Zhigang $\mathrm{Ge}^{1}$, Songyang $\mathrm{Li}^{2}$, Haicheng Wu${ }^{1}$, Lili Wen ${ }^{1}$, Wenming Wang ${ }^{1}$, and Huanyu Zhang ${ }^{1}$ \\ 1 China Nuclear Data Center, China Institute of Atomic Energy, Beijing 102413, China \\ 2 Tsinghua University, Beijing 10086, China
}

\begin{abstract}
China is developing the nuclear data processing code Ruler, which can be used for producing multigroup cross sections and related quantities from evaluated nuclear data in the ENDF format [1]. The Ruler includes modules for reconstructing cross sections in all energy range, generating Doppler-broadened cross sections for given temperature, producing effective self-shielded cross sections in unresolved energy range, calculating scattering cross sections in thermal energy range, generating group cross sections and matrices, preparing WIMS-D format data files for the reactor physics code WIMS-D [2]. Programming language of the Ruler is Fortran-90. The Ruler is tested for 32-bit computers with Windows-XP and Linux operating systems. The verification of Ruler has been performed by comparison with calculation results obtained by the NJOY99 [3] processing code. The validation of Ruler has been performed by using WIMSD5B code.
\end{abstract}

\section{Introduction}

The Ruler is a computer code package for generating multi-group cross sections and related quantities from evaluated nuclear data in the ENDF format.

The objective to create the computer programs for the production of multi-group constants for the calculation of reactor physics using evaluated data was conceived 6 years ago. By that time, the project of the self-reliance development of key design codes for nuclear power plants was created, and the self-reliance development of nuclear data processing code is an essential part one. The Ruler has been developed since then.

The Ruler has been developed through the formula derivation, requirements analysis, system design, detailed design, coding, unit testing, sub-system testing and system testing. The well-proven models and methods for generating multi-group constants are used, including resonance reconstruction, Doppler broadening, thermal scattering processing, group cross section and scattering matrices computing, as well as resonance self-shielding treatment.

The Ruler consists of a set of functional modules, each performing a defined processing task. They are main code, data management modules, physics modules, interface modules. They are linked to one another by input and output files. Each of functional modules is supported by a number of subsidiary modules.

The Ruler is China's first multi-group constants generating code system for reactor physics and shielding design, and the development of Ruler will be continued.

\section{Content of the Ruler}

The Ruler is designed with a modular structure, which contains four functional modules, which are supported by a number of subsidiary modules. They are the main code,

a e-mail: ping@ciae.ac.cn the data management modules, the physics modules, and the interface modules.

\subsection{Architecture of the ruler}

For efficient programming, we used the independent ENDF I/O system in the development of the Ruler code, that the data input and output module is separated from the computation modules in the Ruler.

The Ruler has four functional modules, which consists of 11 subsidiary modules, they are the main driver module, data input and output module, public mathematics module, system common subroutine module, data mode converting module, resonance reconstruction module, Doppler broadening module, the unresolved resonance selfshielding module, thermal data generating module, neutron multi-group constants producing module, WIMS-D format data library preparing module, respectively.

Figure 1 shows the architecture of Ruler. The data management modules, the physics modules and the interface modules can be used with relatively independence. Those modules can access the PENDF files obtained by other processing code.

\subsection{The endf input-output system}

The Ruler has independent ENDF I/O system, which consists of two parts. They are the data type and user interface. The data type includes all ENDF files, such as cross sections, angular distribution etc. The user interface includes reading ENDF files and writing ENDF files etc.

Table 1 gives the user interface and function of ENDFIO.

The basic concept of ENDF I/O system is packaging the decentralized I/O operation into a library that programming-maker can use API (Application Programming Interface)-Centric programming pattern to replace the File-centric programming pattern of NJOY. 


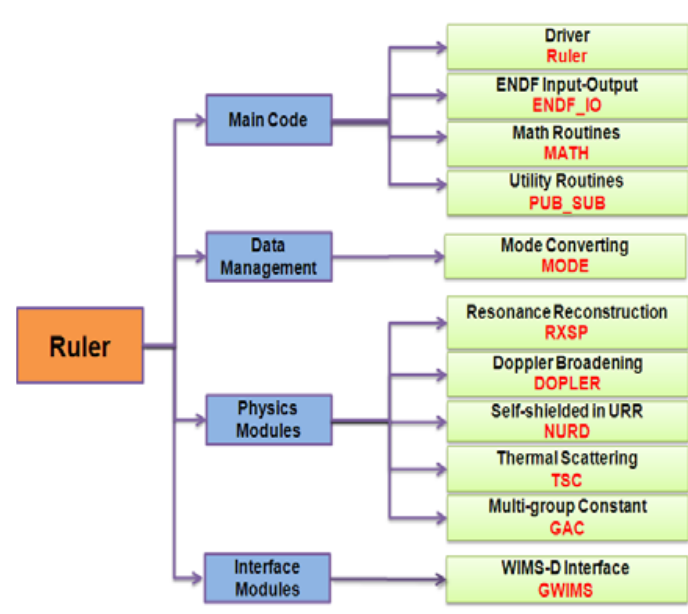

Figure 1. Structure of the Ruler.

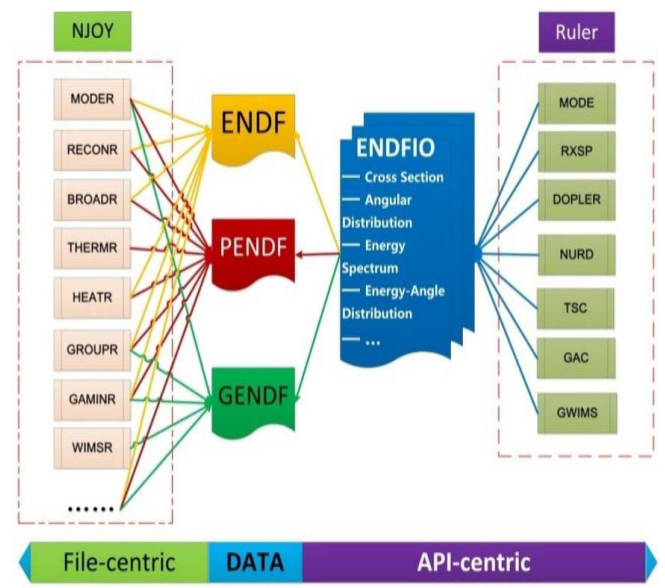

Figure 2. Different programming patterns between Ruler and NJOY.

Table 1. User interface and function of ENDF_IO.

\begin{tabular}{|c|c|}
\hline Interface & Function \\
\hline $\begin{array}{c}\text { Subroutine } \\
\text { read_endf_file(fin,endf,mat) }\end{array}$ & $\begin{array}{c}\text { Read data from } \\
\text { (P)ENDF files }\end{array}$ \\
\hline $\begin{array}{c}\text { Subroutine } \\
\text { read_gendf_file(fin,endf,mat) }\end{array}$ & $\begin{array}{c}\text { Read data from } \\
\text { GENDF files }\end{array}$ \\
\hline $\begin{array}{c}\text { Subroutine } \\
\text { write_endf_file(fout,endf, } \\
\text { overwrite) }\end{array}$ & $\begin{array}{c}\text { Write onto a } \\
\text { (P)ENDF files }\end{array}$ \\
\hline $\begin{array}{c}\text { Subroutine } \\
\text { write_gendf_file(fout,endf, } \\
\text { overwrite) }\end{array}$ & $\begin{array}{c}\text { Write onto a } \\
\text { GENDF files }\end{array}$ \\
\hline Subroutine del_endf(endf) & Release memory space \\
\hline Subroutine del_gendf(gendf) & Release memory space \\
\hline Interface insert_mt & $\begin{array}{c}\text { Insert file onto } \\
\text { specified position }\end{array}$ \\
\hline Interface find_mt & Find specified MT file \\
\hline
\end{tabular}

Figure 2 shows the comparison of programming patterns between Ruler and NJOY.

The use of independent ENDF I/O system in the Ruler results that the Ruler is much more advanced in the aspects of the running time, the maintainability and the extendibility, especially is easy for modification while the ENDF format is changed.

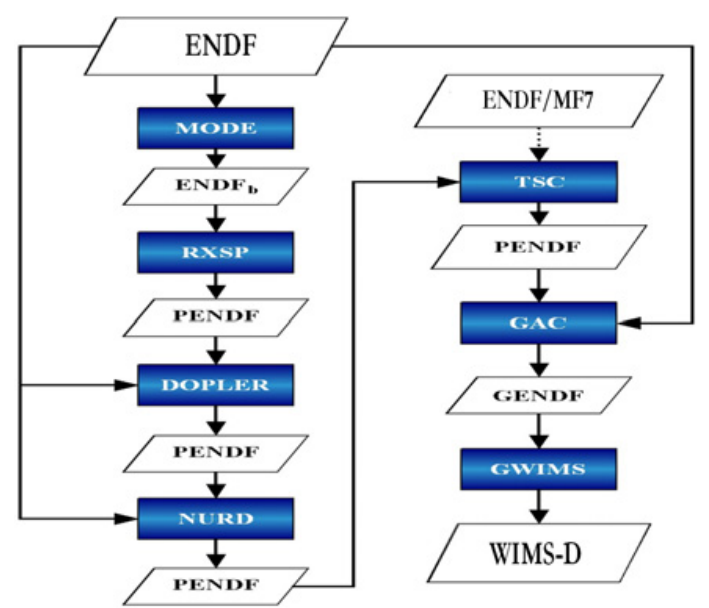

Figure 3. Flow diagram of the Ruler.

\subsection{Flow diagram of the ruler}

The Ruler can be used for generating the WIMS-D format data files from ENDF format files. Figure 3 shows the flow diagram of the Ruler.

The run of the Ruler begins with the reading of ENDF format files. Then, a successive data processing for each module is carried out.

The following brief summary illustrates the general flow of data processing in the Ruler.

MODE converts ENDF library files for binary mode for calculation efficiency.

RXSP reads an ENDF file and produces a common energy grid for all reactions. Resonance cross sections are reconstructed in all energy range. The resulting pointwise cross sections are written onto a "point-ENDF" (PENDF) file for future use.

DOPLER reads a PENDF file and Doppler-broadens the data using the accurate point-kernel method to generate Doppler-broadened cross sections for given temperature. The results are written out on a new PENDF file for future use.

NURD produces effective self-shielded pointwise cross sections, versus temperatures and background cross sections in the unresolved range. This is done for each temperature produces by DOPLER. The results are added to the PENDF file.

TSC produces scattering cross sections in the thermal range. The results are added to the PENDF file.

GAC processes the pointwise cross sections into multigroup form. The weighting function for group averaging can be taken to be the Bondarenko form, or it can be computed from the slowing-down equation for a heavy absorb in a light moderator. The results are saved on a GENDF file.

GWIMS prepares libraries for the thermal reactor assembly code WIMS-D.

\section{Verification and validation of Ruler}

The verification and validation of Ruler has been performed by comparison with the calculation results obtained by NJOY99 code. 


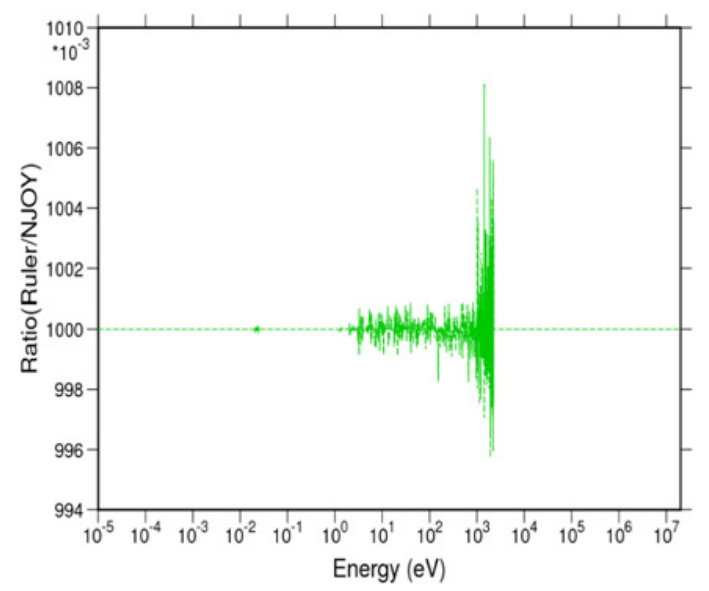

Figure 4. Reconstruction fission cross sections for U-235.

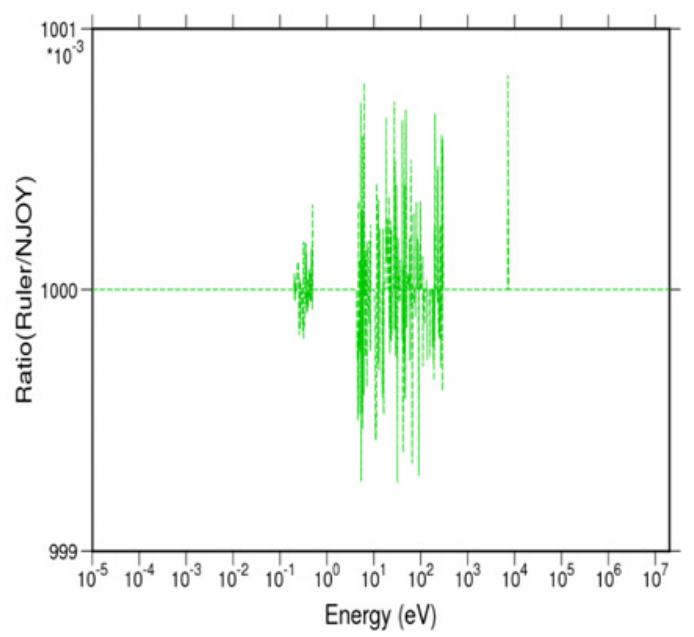

Figure 5. Reconstruction fission cross sections for $\mathrm{Pu}-241$.

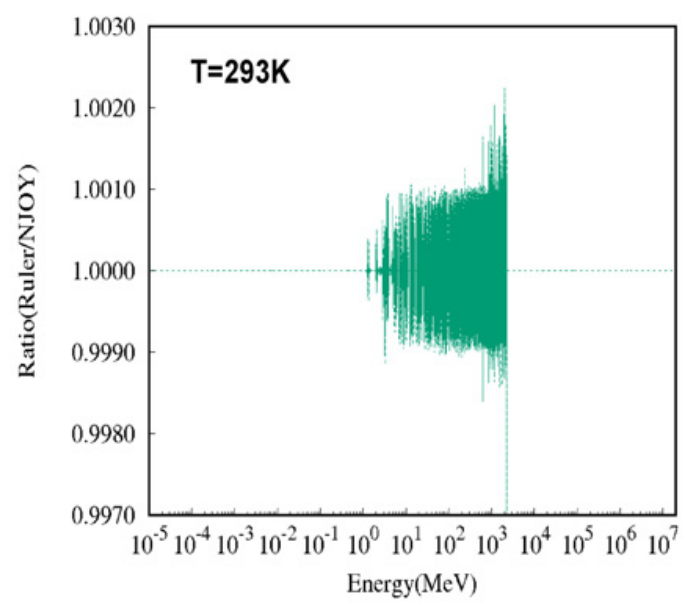

Figure 6. Doppler broadening fission cross sections for U-235.

\subsection{Comparison of calculated cross sections}

Figures 4 to 11 show the comparison of cross sections for some materials between Ruler and NJOY. It can be seen that the reconstruction cross sections of Ruler show agreement with NJOY99 within 1\%, Doppler broadening cross sections of Ruler show agreement with NJOY99 within 3\%, group averaging cross sections are similar for

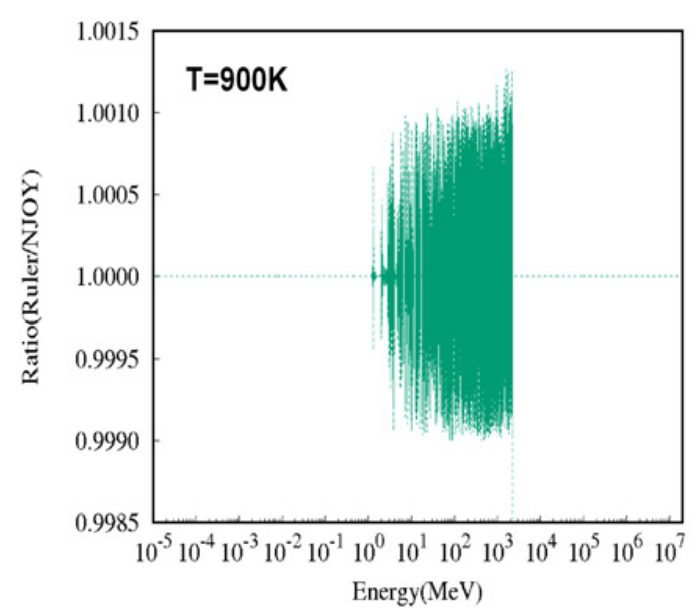

Figure 7. Doppler broadening fission cross sections for U-235.

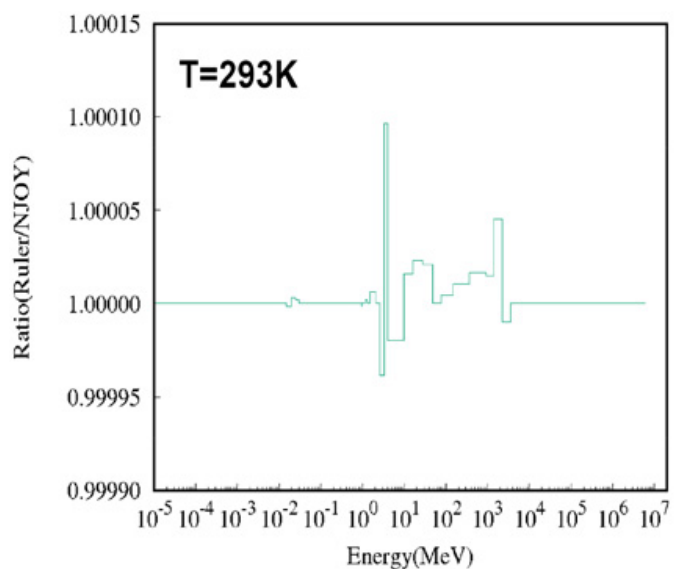

Figure 8. 69 groups averaged fission cross sections for U-235.

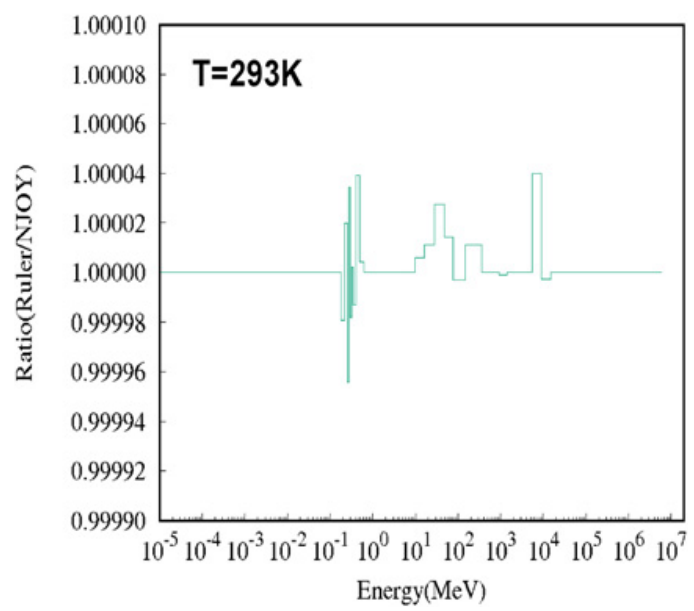

Figure 9. 69 groups averaged fission cross sections for $\mathrm{Pu}-241$.

Ruler and NJOY, and the WIMS-D format cross sections of Ruler show agreement with NJOY99 within $1 \%$.

\subsection{Comparison of running time}

Table 2 shows the comparison of running time for generating a WIMS-D format data file between Ruler and NJOY99. It can be seen that Ruler has less computing time compared with NJOY99. This is because of the independent ENDF I/O system in the Ruler that read-once, 


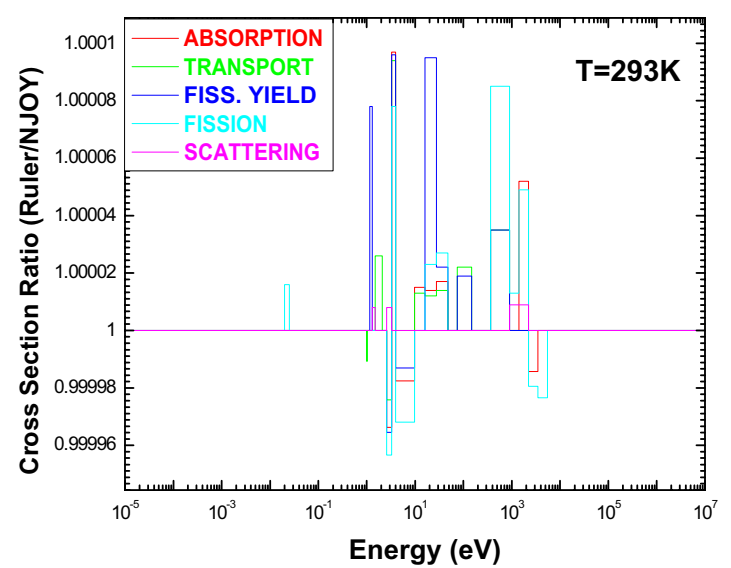

Figure 10. WIMS-D 69 groups cross sections for U-235.

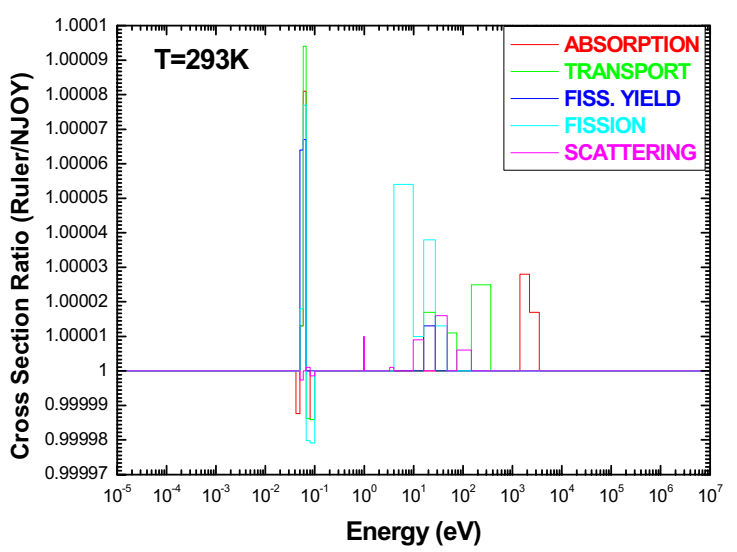

Figure 11. WIMS-D 69 groups cross sections for Pu-239.

Table 2. Comparison of running time.

\begin{tabular}{|l|l|l|l|}
\hline \multirow{2}{*}{ Nuclide } & \multicolumn{2}{|l|}{ Running time (s) } & Speed-up ratio \\
\cline { 2 - 4 } & NJOY & Ruler & NJOY/Ruler \\
\hline 1-H-H ${ }_{2} \mathrm{O}$ & 152.7 & 104.2 & 1.47 \\
\hline $1-\mathrm{H}-\mathrm{ZrH}$ & 410.2 & 365.9 & 1.12 \\
\hline 1-D-D $\mathrm{D}_{2} \mathrm{O}$ & 157.6 & 101.6 & 1.55 \\
\hline 6-C-nat & 84.4 & 61.7 & 1.37 \\
\hline $8-\mathrm{O}-16$ & 25.9 & 14.9 & 1.74 \\
\hline $13-\mathrm{Al}-27$ & 7.0 & 4.3 & 1.63 \\
\hline 26-Fe-56 & 23 & 19.9 & 1.16 \\
\hline 36-Kr-83 & 5.7 & 3.1 & 1.84 \\
\hline $42-\mathrm{Mo}-95$ & 7 & 5.7 & 1.23 \\
\hline 92-U-235 & 247.3 & 187.0 & 1.32 \\
\hline 92-U-238 & 448.6 & 225.3 & 1.99 \\
\hline 94-Pu-239 & 136.7 & 67 & 2.04 \\
\hline
\end{tabular}

use-many in the Ruler, but read-many, use-many in the NJOY.

\subsection{Benchmark tests for Ruler}

Benchmark tests for Ruler have been performed using the WIMSD5B code. The ENDF files are from ENDF/ B-VII.1 [4]. All benchmarks are from WLUP/IAEA [5]. The WIMS-D format data files were generated with Ruler according to the flow diagram of Fig. 2 .

Figures 12 to 13 show the comparison of benchmarking results between Ruler, NJOY and experiments. The results of NJOY are from WLUP/IAEA [6]. It can

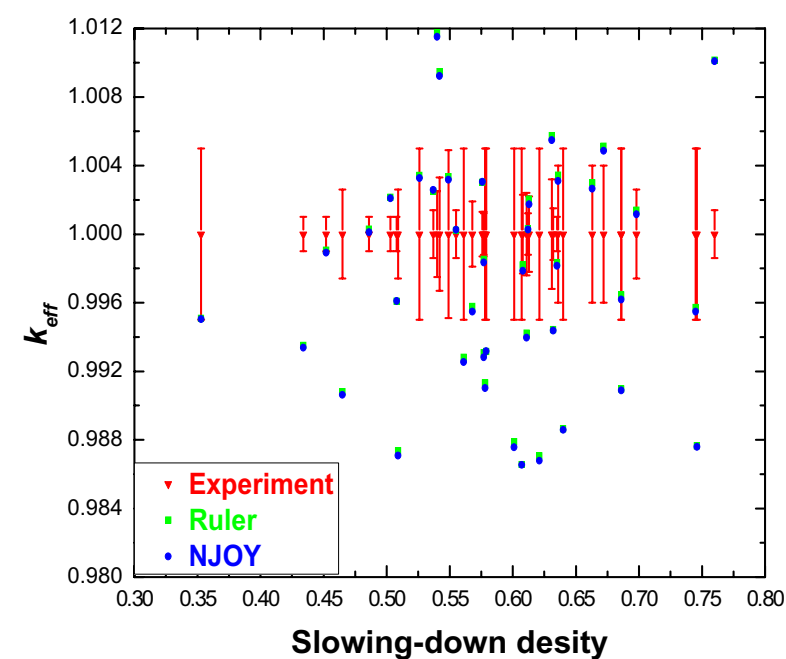

Figure 12. $\mathrm{H}_{2} \mathrm{O}$ moderated $\mathrm{UO}_{2}$ lattices $k_{\text {eff }}$ vs. q. Enrichment ( $>2$ and $<4$ wt $\% \mathrm{u}-235$ ).

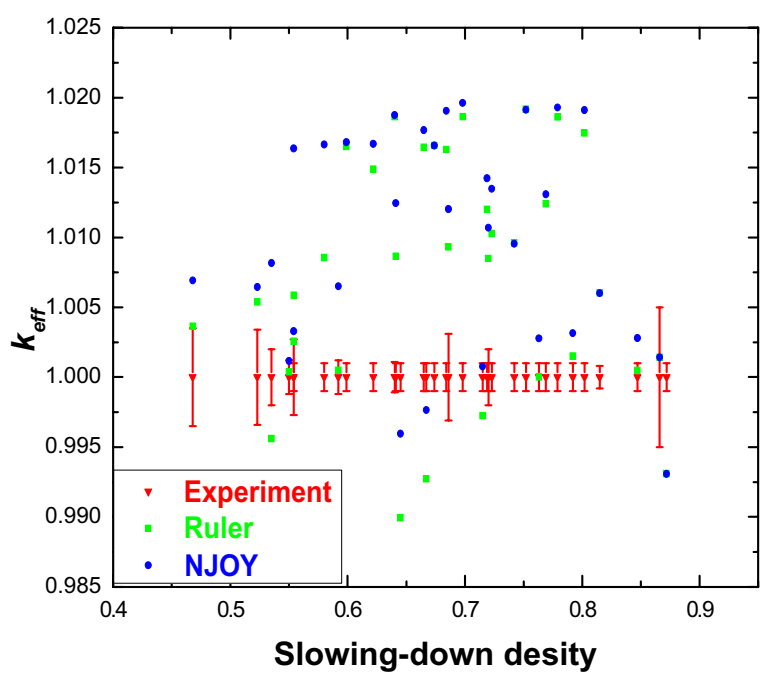

Figure 13. $\mathrm{H}_{2} \mathrm{O}$ moderated MOX lattices $k_{e f f}$ vs. q. Enrichment ( $>2 \mathrm{wt} \%$ fissile metal).

be seen that the calculated results of Ruler show good agreement with those of NJOY99 for $\mathrm{H}_{2} \mathrm{O}$ moderated uranium oxide lattices. All values of $k_{\text {eff }}$ generated with Ruler are slightly lower than the corresponding values obtained with NJOY $99 \mathrm{H}_{2} \mathrm{O}$ moderated MOX lattices, with similar tread for Ruler and NJOY.

\section{Conclusions and intentions}

The primary objective to create nuclear data processing code has been accomplished. WIMS-D format cross sections and other related quantities were generated using Ruler. The verification of Ruler has been performed by comparison with calculation results obtained by NJOY99.396 processing code. The benchmark tests for the library prepared by Ruler have been performed with WIMSD5B code.

The future development of Ruler will be continued, which includes photon processing module and more interface modules. The modification of ENDF_IO module for new data format will be considered. 


\section{References}

[1] M. Herman and A. Trkov, Eds., ENDF-6 Formats Manual, Data Formats and Procedures for the evaluated nuclear data File ENDF/B-VI and ENDF/B-VII, Brookhaven National Laboratory report BNL-903652009 (ENDF-102) (June 2009). For the latest version of this report, see http//www.nndc.bnl.gov and take the ENDF tab to ENDF-6 formats Manual

[2] WIMS-D5, OECD/NEA Data Bank Documentation, Package ID No. 1507/02 (1998), http//www.nea.fr/html/dbprog/
[3] R. E. MacFarlane and D. W. Muir. NJOY99.0, Code System for Producing Pointwise and Multigroup Neutron and Photon Cross Sections from ENDF/B Data. Los Alamos National Laboratory Report, LA-12740-M, 2000

[4] M. B. Chadwick et al., "ENDF/B-VII.1 Nuclear Data for Science and Technology : Cross sections, Covariances, Fission Product Yields and Decay Data," Nuclear Data Sheets 112, 2887-2996 (2011)

[5] http://www-nds.iaea.org/wimsd/benchmarks . htm

[6] http://www-nds.iaea.org/wimsd/results / endfb7.1st 